\title{
Tetracene Formation by On-Surface Reduction Supporting Information
}

\author{
Justus Krüger, ${ }^{\dagger}$ Niko Pavliček, $¥$ José M. Alonso,§ Dolores Pérez,§ Enrique Guitián,§ \\ Thomas Lehmann, † Gianaurelio Cuniberti,, ,l André Gourdon,\# Gerhard Meyer, $\neq$ Leo \\ Gross, ${ }^{\ddagger}$ Francesca Moresco, ${ }^{\dagger}$ and Diego Peña§ \\ †Institute for Materials Science, Max Bergmann Center of Biomaterials, and Center for Advancing Electronics Dresden, TU Dresden, \\ 01069 Dresden, Germany \\ fIBM Research-Zurich, 8803 Rüschlikon, Switzerland \\ §Centro de Investigación en Química Biolóxica e Materiais Moleculares (CIQUS) and Departamento de Química Orgánica, Universidade \\ de Santiago de Compostela, Santiago de Compostela 15782, Spain \\ \#Centre d'élaboration de matériaux et d'études structurale (CEMES), UPR 8011 CNRS, Nanosciences Group, 29 Rue J. Marvig, P.O. Box \\ 94347, 31055 Toulouse, France \\ "Dresden Center for Computational Materials Science (DCMS), TU Dresden, 01069 Dresden, Germany
}

\section{Table of Contents}

1. Synthesis of diepoxytetracenes syn/anti-1

2. Additional STM/AFM data

References 


\section{Synthesis of diepoxytetracenes syn/anti-1}

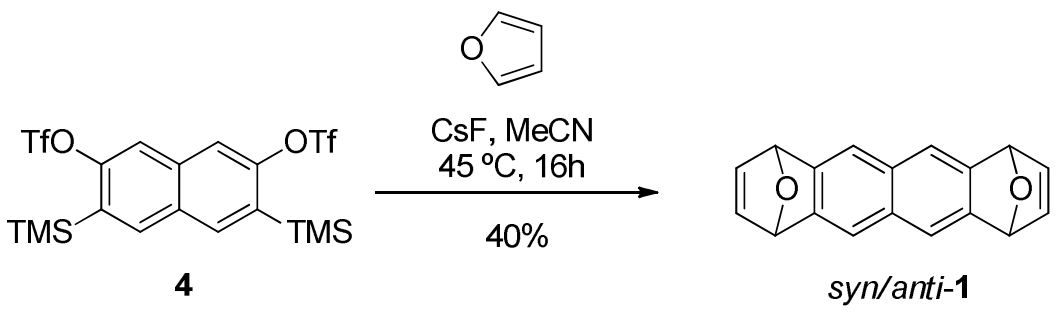

CsF (642 mg, $4.23 \mathrm{mmol}$ ) was added over a solution of bisaryne precursor $4^{1}(300 \mathrm{mg}, 0.53 \mathrm{mmol})$ and furan $(3.0$ $\mathrm{mL}, 42.3 \mathrm{mmol})$ in dry $\mathrm{MeCN}(5.1 \mathrm{~mL})$. The resulting mixture was stirred $16 \mathrm{~h}$ at $45^{\circ} \mathrm{C}$. After evaporation of the solvent under reduced pressure, the residue was purified by column chromatography (hexane/AcOEt 4:1) yielding a mixture of syn- and anti-1,4,7,10-tetrahydro-1,4:7,10-diepoxytetracene (1) as a white solid (56 mg, 40\%). ${ }^{1} \mathrm{H}$ NMR showed the presence of the two non-separable isomers in a 1:1.7 ratio. ${ }^{2}$

${ }^{1} \mathrm{H} \mathrm{NMR}\left(\mathrm{CDCl}_{3}, 300 \mathrm{MHz}\right): \delta 5.78(8 \mathrm{H}, \mathrm{s}), 6.95$ (4H, br s, major isomer), 6.98 (4H, br s, minor isomer), 7.49 (4H, s, minor isomer), 7.50 (4H, s, major isomer). EM (El+), m/z (\%): $260(\mathrm{M}+1,19), 201$ (100). M. P. >250 $\mathrm{C}$.
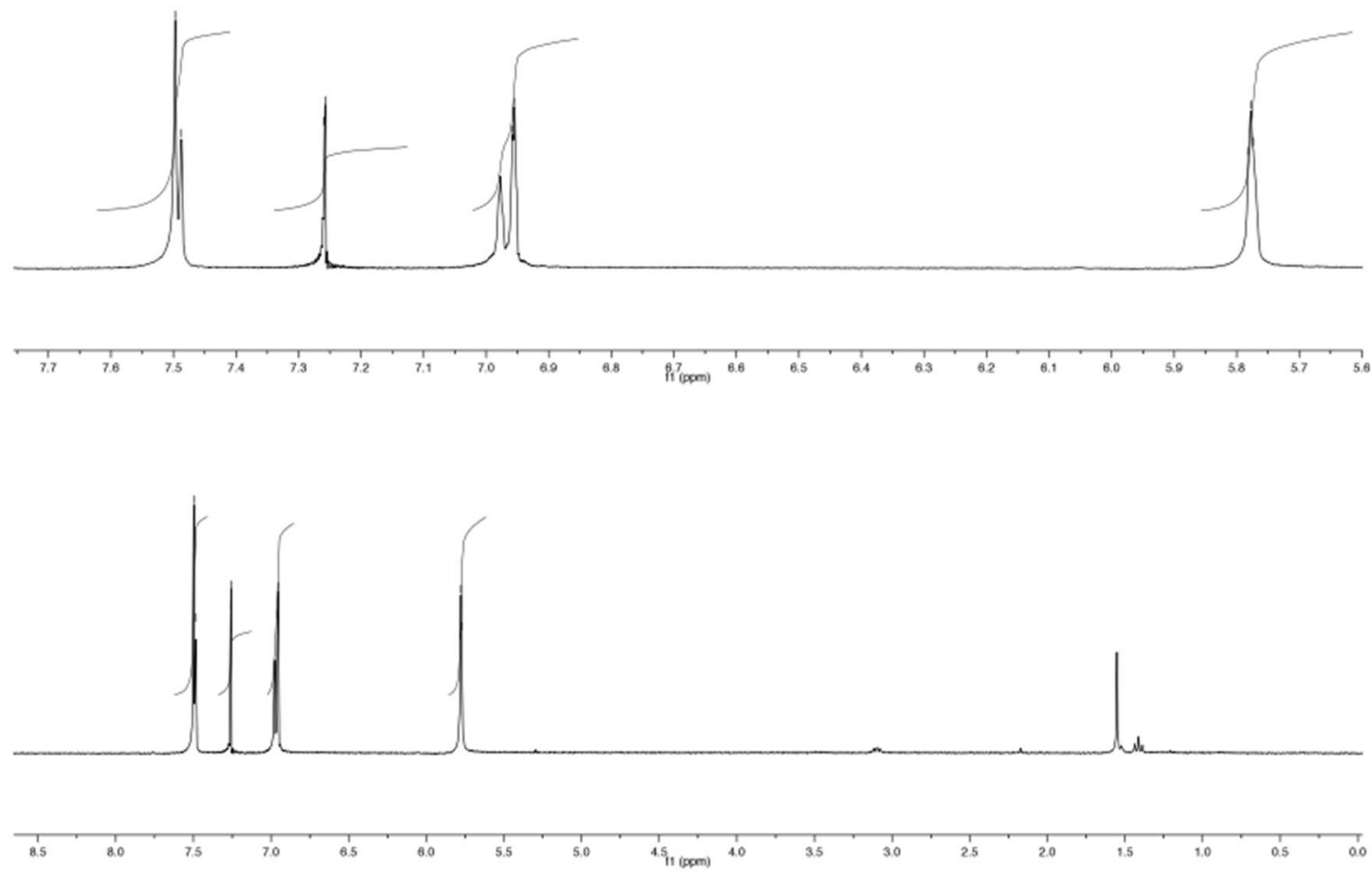

Figure S1. ${ }^{1} \mathrm{H}$ NMR of the mixture syn/anti-1 in $\mathrm{CDCl}_{3}$. 


\section{Additional STM/AFM data}

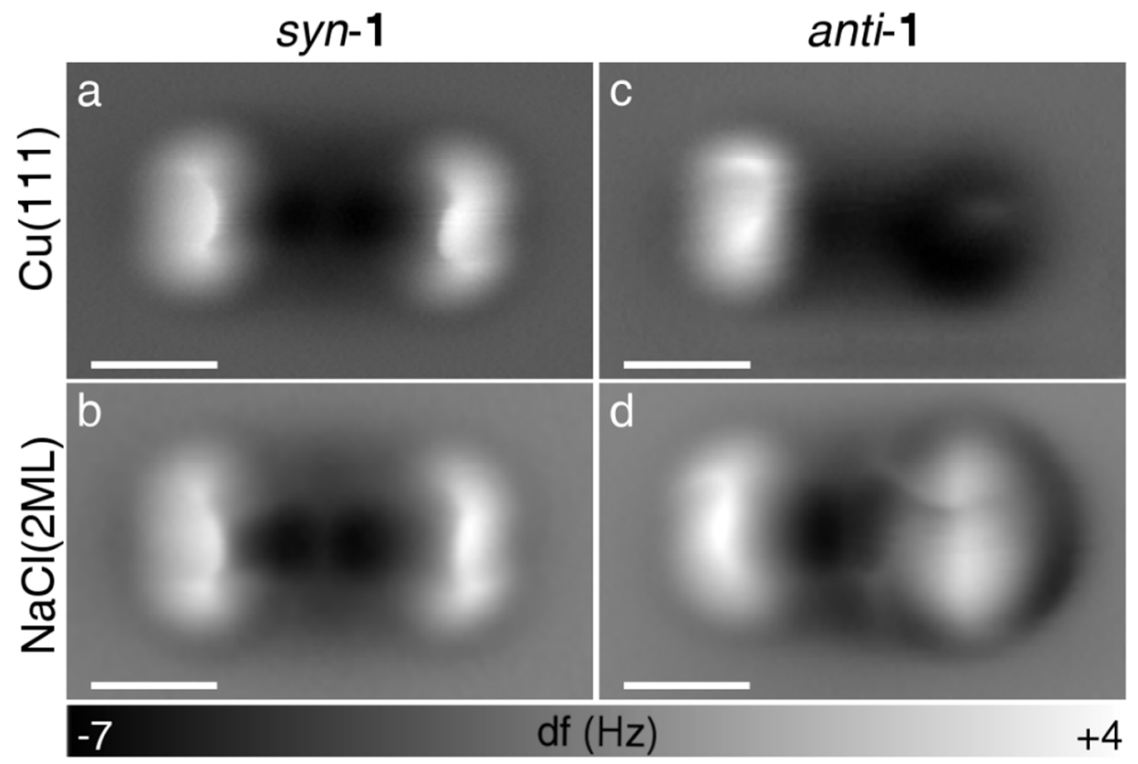

Figure S2. Raw AFM frequency shift data of syn/anti-1 on $\mathrm{Cu}(111)$ and $\mathrm{NaCl}(2 \mathrm{ML})$, respectively. All scale bars refer to $5 \AA$. Measurements were acquired in constant-height modus at $V=0 \mathrm{~V}$ with a positive offset $\Delta z$ corresponding to a distance decrease with respect to an STM set-point current of $\mathrm{I}=2 \mathrm{pA}$ at $\mathrm{V}=0.1 \mathrm{~V}$ above the clean $\mathrm{Cu}(111)$ and $\mathrm{NaCl}(2 \mathrm{ML})$ surfaces, respectively. (a) syn-1 on $\mathrm{Cu}(111)$ with $\Delta \mathrm{z}=+0.4 \AA$ (b) syn-1 on $\mathrm{NaCl}(2 \mathrm{ML})$ with $\Delta \mathrm{z}=-2.4 \AA$ (c) anti-1 on $\mathrm{Cu}(111)$ with $\Delta \mathrm{z}=-0.3 \AA$ (d) anti-1 on $\mathrm{NaCl}(2 \mathrm{ML})$ with $\Delta \mathrm{z}=-1.6 \AA$.

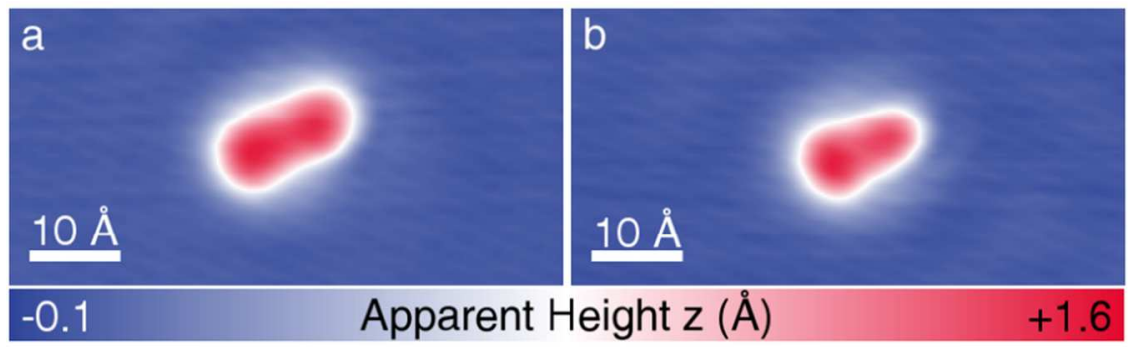

Figure S3. Constant-current STM images of syn-1 (a) and anti-1 (b) on $\mathrm{Cu}(111)$. Scanning conditions: $V=100 \mathrm{mV}$ and $\mathrm{I}=2 \mathrm{pA}$. 


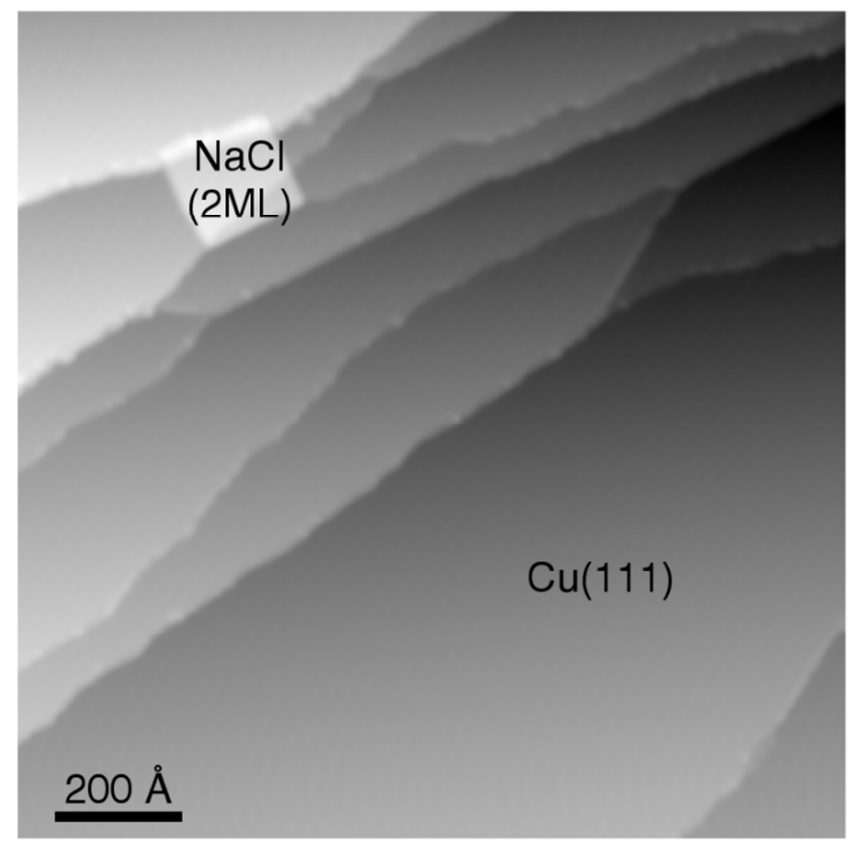

Figure S4. STM Overview scan after annealing the surface up to $120^{\circ} \mathrm{C}$. The sample terraces are mostly empty and only the Cu edges feature a decoration. Scanning conditions: $V=500 \mathrm{mV}$ and I $=2 \mathrm{pA}$.

\section{References}

1. Kitamura, C.; Abe, Y.; Ohara, T.; Yoneda, A.; Kawase, T.; Kobayashi, T.; Naito, H.; Komatsu, T. Synthesis and Crystallochromy of 1,4,7,10-Tetraalkyltetracenes: Tuning of Solid-State Optical Properties of Tetracenes by Alkyl Side-Chain Length. Chem. Eur. J. 2010, 16, 890-8.

2. Gribble, G. W.; Perni, R. B.; Onan, K. D. Twin Benzannulation of Naphthalene Via 1,3-, 1,6-, and 2,6Naphthodiyne Synthetic Equivalents. New Syntheses of Triphenylene, Benz[a]Anthracene, and Naphthacene. J. Org. Chem. 1985, 50, 2934-2939. 